Pacific Journal of Mathematics

ROOT LOCOLOGIES AND IDEMPOTENTS OF LIE AND 


\section{ROOT LOCOLOGIES AND IDEMPOTENTS OF LIE AND NONASSOCIATIVE ALGEBRAS}

\section{DAVID J. WINTER}

Locological spaces are introduced. The $G$-locology for a subset $R$ of a group $G$ leads to the symmetric $G$-topology of $R$. The connected components of $R$ correspond to ideals of any normal finite dimensional $G$-graded nonassociative algebra $A$ which, for $A$ an idempotent Lie algebra with set $R$ of roots, are the central primitive idempotents of $A$.

o. Introduction. The underlying ideas in this paper are that "ideals" in a Lie algebra or graded nonassociative algebra $A$ correspond to "open sets" in the set $R$ of roots of $A$; and "direct sums" correspond to "disjoint unions of open sets."

The first section is devoted to making these ideas precise, in the language of locologies and topologies for $R$.

The second section is devoted to the development of a theory of decompositions of idempotent nonassociative algebras 1 as sums $1=E_{1}+\cdots+E_{n}$ of pairwise orthogonal central primitive idempotents; and to showing for idempotent Lie algebras that the central primitive idempotents correspond to the connected components $R_{1}, \cdots, R_{n}$ of $R$ discussed in Section 1 .

The third section is devoted to relating the open set structure of $R$ to the ideal structure of a Lie algebra $L$ not assumed to be idempotent, taking as starting point Theorem 1.21.

1. Locological spaces and root locologies. Let $R$ be a set, $k$ a set with a specified point $0 \in k$ called the origin of $k, H$ a collection of functions from $R$ into $k$. Suppose that $H$ contains the zero function which maps all elements of $R$ into 0 . Suppose, furthermore, that for each $\mathrm{a} \in R, x(a) \neq 0$ for some $x \in H$. For $X \subset H$, let $R(X)=\{a \in R \mid x(a)=0$ for all $x \in X\}$. Then the collection $\mathscr{C}=\{R(X) \mid X \subset H\}$ of subsets of $R$ contains $R$ and $\phi$; and is closed under intersections since

$$
R\left(\bigcup_{i \in I} X_{i}\right)=\bigcap_{i \in I} R\left(X_{i}\right) .
$$

We call $R(X)$ the locus of zeros of $X$. The collection $\mathscr{C}$ is a locology for $R$ in the sense of the following definition.

Definition 1.1. A locology for a set $R$ is a collection $\mathscr{C}$ of subsets of $R$ such that 
(1) $\phi \in \mathscr{C}$ and $R \in \mathscr{C}$;

(2) $\mathscr{C}$ is closed under intersections, that is, $\mathscr{S} \subset \mathscr{C}$ implies $\bigcap_{s \in \mathscr{S}} S \in \mathscr{C}$.

A locological space is a set $R$ together with a locology $\mathscr{C}$ for $R$.

If, in the above example, $H$ also separates the points of $R$, we can imbed $R$ in the set $F(H, k)$ of functions from $H$ to $k$ by regarding $a \in R$ as the function $a: H \rightarrow k$ such that $a(x)=x(a)$ for $x \in H$. Thus, $R(X)$ so imbedded is $R(X)=\{a \in R \mid a(x)=0$ for all $x \in X\}$. Let us suppose furthermore that $k$ is a group with product + (not necessarily commutative) and identity equal to the origin 0 . Then the sets $R(X)$ satisfy the following conditions, $a+b$ and $-a$ denoting pointwise product and inverse of $a, b \in R$ and $a \in R$ respectively.

(1) if $a, b \in R(X)$, then $a+b \in R(X)$ if $a+b \in R, a-b \in R(X)$ if $a-b \in R$, and $(-a)+b \in R(X)$ if $(-a)+b \in R$;

2. if $a \in R(X)$ and $-a \in R$, then $-a \in R(X)$.

Thus, $R(x)$ is closed and symmetric in the $G$-locology for $R$ in the sense of the following definition, $G$ being the group $G=F(R, k)$.

Definition 1.2. Let $R$ be subset of a group $G$ with product $a b(a, b \in G)$. Then a subset $S$ of $R$ is $G$-closed if $\left(S^{2} \cup S S^{-1} \cup S^{-1} S\right) \cap$ $R \subset S$, and $S$ is symmetric if $S^{-1} \cap R \subset S$. Here, $S T=\{a b \mid a \in S$, $b \in T\}, S^{2}=S S, S^{-1}=\left\{a^{-1} \mid a \in S\right\}$ for $S, T \subset G$. The collection $\mathscr{C}$ of $G$-closed (respectively symmetric $G$-closed) subsets of $R$ is called the G-locology (respectively symmetric G-locology) of $R$.

The G-locology (respectively symmetric G-locology) for a subset $R$ of a group $G$ obviously satisfies the axioms for a locology for $R$.

We now assume that $R$ is an arbitrary locological space with locology $\mathscr{C}$. The elements of $\mathscr{C}$ are called the closed sets of $R$, their complements the open sets of $R$. Note that $R$ and $\phi$ are both open and closed. For any subset $S$ of $R, \mathscr{C}_{S}=\{A \cap S \mid A \in \mathscr{C}\}$ is a locology for $S$, called the relative locology on $S$. The closed and open sets of $S$ are called the relatively closed and open sets of $S$ respectively. If $S$ is closed, $\mathscr{C}_{S}=\{A \in \mathscr{C} \mid A \subset S\}$. The closure of a subset $S$ of $R$ is the intersection $\bar{S}$ of all closed sets of $R$ containing $S$. Note that $\bar{S}$ is closed, contains $S$ and is contained in every closed set containing $S$. We say that a subset $S$ of $R$ is connected if $S=S_{1} \cup S_{2}$ where $S_{1}$ and $S_{2}$ are disjoint and relatively closed in $S$ implies that $S=S_{1}$ or $S=S_{2}$.

Proposition 1.3 Let $S$ be connected. Then $\bar{S}$ is connected.

Proof. For $A, B$ closed, $\bar{S} \subset A \cup B$ and $\bar{S} \cap A \cap B=\phi$, we must 
show that $\bar{S} \subset A$ or $\bar{S} \subset B$. But this follows from the fact that $A$ and $B$ are closed and, since $S$ is connected, $S \subset A$ or $S \subset B$.

For $x \in R, C(x)$ is the union of all connected subsets of $R$ which contain $x$.

TheOREM 1.4. For $x \in R, C(x)$ is closed and connected and contains $x$. For $x, y \in R$, either $C(x)=C(y)$ or $C(x) \cap C(y)=\phi$.

Proof. Since $\{x\}$ is connected, $C(x)$ contains $x$. Suppose that $C(x) \subset A \cup B$ and $C(x) \cap A \cap B=\phi$ with $A, B$ closed. We may assume with no loss of generality that $x \in A$. Then every connected set $S$ containing $x$ is contained in $A$, so that $C(x) \subset A$. Thus, $C(x)$ is connected. Since $\overline{C(x)}$ is connected, $\overline{C(x)}=C(x)$ and $C(x)$ is closed. Suppose that $C(x) \cap C(y) \cdot \ni z$. Then $C(z) \supset C(x), C(z) \supset C(y)$, whence $C(x)=C(z)=C(y)$.

The above theorem shows that the sets $C(x)$ are the maximal connected subsets of $R$. We call $C(x)$ the connected component of $R$ containing $x$.

CoRollary 1.5. $R$ can be decomposed as a disjoint union $R=$ $\mathrm{U}_{i \in I} R_{i}$ where the $R_{i}(i \in I)$ are the connected components of $R$.

The connected components $R_{i}$ of $R$ are closed.

CoROLlaRY 1.6. Suppose that $R=\bigcup_{i \in I} R_{i}$ (disjoint union) where $R_{i}$ is nonempty, open and connected for all $i$. Then

(1) the $R_{i}$ are the connected components of $R$;

(2) each open and closed subset $S$ of $R$ is a union $S=\bigcup_{i \in I} R_{i}$ of certain of the $R_{i}$; and every such union is open and closed.

In particular, the collection $\mathscr{D}$ of open and closed subsets of $R$ is closed under unions and intersections and is therefore a topology for $R$.

Proof. We first prove part of (2), namely, that each union $S=\bigcup_{i \in I} R_{i}$ of a subcollection $R_{i}(i \in I)$ of the $R_{i}$ is open and closed. Since the $R_{i}$ are open, $S$ is open since $S^{c}=\bigcap_{i \in I} R_{i}^{i}$ is closed-as the intersection of closed sets. Similarly, $S^{c}=\mathrm{U}_{j \notin I} R_{j}$ is open. Thus, $S$ is also closed. Taking $I=\{i\}$, we have shown in particular that each $R_{i}$ is open and closed, as is its complement $R_{i}^{c}$ in $R$. This having been shown, we now note that for (1), it suffices to show that $C=R_{i}$ for any connected set $C$ containing $R_{i}$. This follows 
easily from the connectedness of $C$ and the fact that $R_{i}, R_{i}^{c}$ are closed and disjoint, $C \subset R_{i} \cup R_{i}^{c}$ and $C \cap R_{i}$ is nonempty. For the remaining direction of (2), it suffices to show that whenever $R_{i} \cap S \neq \phi, S$ contains $R_{i}$. This follows directly from the fact that $S, S^{c}$ are closed and disjoint, $R_{i}$ is connected, $R_{i} \subset S \cup S^{c}$ and $R_{i} \cap$ $S \neq \phi$.

We now specialize our considerations to a fixed subset $R$ of a group $G$. We regard $R$ as locological space with the $G$-locology for $R$, and refer to $R$ with this locology as a G-locological space. For $S \subset R$, we denote the complement of $S$ in $R$ by $S^{c}$. We say that $S$ is G-open if $S^{c}$ is $G$-closed.

\section{TheOREM 1.7. Let $S$ be a G-closed set of $R$. Then}

$\left(S S^{c} \cup S^{c} S \cup S^{-1} S^{c} \cup S^{c} S^{-1} \cup S\left(S^{c}\right)^{-1} \cup\left(S^{c}\right)^{-1} S\right) \cap R \subset S^{c}$

Proof. Let $a \in S, b \in S^{c}$. Then we have $b=a^{-1}(a b)=(b a) a^{-1}=$ $a\left(a^{-1} b\right)=\left(b a^{-1}\right) a=\left(a b^{-1}\right) a^{-1}=a\left(b^{-1} a\right)^{-1}$. Let $d$ be any one of the elements $a b, b a, a^{-1} b, b a^{-1}, a b^{-1}, b^{-1} a$. Since $S$ is closed, $b \notin S$ and $b \in\left(S^{-1} d \cup d S^{-1} \cup S d \cup d S \cup d^{-1} S \cup S d^{-1}\right)$, it follows that $d \notin S$. Thus, $d \in R$ implies $d \in S^{c}$.

In general, the collection $\mathscr{D}$ of open and closed sets in a locological space $R$ is not closed under finite unions and intersections. For example, if $R$ is the disjoint union of nonempty sets $A, B, C, D$, then $\mathscr{D}=\left\{\phi, R, A, B, A^{c}, B^{c}\right\}$ where the closed sets of $R$ are $\phi, R$, $A, B, C, D, A^{c}, B^{c},(A \cup B)^{c}$. However, $\mathscr{D}$ is closed under finite unions and intersections for $G$-locological spaces $R$.

THEOREM 1.8. Let $\mathscr{D}$ be the collection of subsets $S$ of $R$ which are both G-open and G-closed. Let $S, T \in \mathscr{D}$. Then

(1) for $a \in S, b \notin S$, we have $a b \notin R, a^{-1} b \notin R, a b^{-1} \notin R$;

(2) $S \cup T$ and $S \cap T$ are in $\mathscr{D}$.

Proof. (1) follows from Theorem 1.7 because, since $S$ and $S^{c}$ are both closed, we have $\left(S S^{c} \cup S^{-1} S^{c} \cup S S^{c^{-1}}\right) \cap R \subset S \cap S^{c}=\phi$. For (2), it suffices to prove that $S \cup T$ is closed and open for all $S, T \in$ $\mathscr{D}$, since $S \in \mathscr{D}$ implies $S^{c} \in \mathscr{D}$ and $(S \cap T)^{c}=S^{c} \cup T^{c}$. Moreover, $S \cup T$ is clearly open, since $S$ and $T$ are open. We claim that $S \cup T$ is closed. Thus, let $a, b \in S \cup T$. Then one of the following cases result:

(1) $(a, b \in S)$ or $(a, b \in T)$;

(2) $(a \in S, a \notin T, b \in T, b \notin S)$ or $(b \in S, b \notin T, a \in T, a \notin S)$. 
In case (1), $\left\{a b, a^{-1} b, a b^{-1}\right\} \cap R \subset S \cup T$. In case (2), the same is true by the first assertion of the theorem which we have already proved.

Corollary 1.9. Let $S, T \in \mathscr{D}$ and let $a \in S, b \in T$. Then either $a, b \in S \cap T$ or $R$ contains none of the elements $a b, a^{-1} b, a b^{-1}$.

Proof. Suppose that $S \cap T$ does not contain both of $a, b$. Then either $a \in S$ and $b \notin S$ or $a \notin T$ and $b \in T$. In either case, $a b \notin R$, $a^{-1} b \notin R$ and $a b^{-1} \notin R$ by Theorem 1.8 .

Corollary 1.10. If $\mathscr{D}$ is finite, then $R=R_{1} \cup \cdots \cup R_{n}$ (disjoint union) where the $R_{i}$ are the minimal nonempty elements of $\mathscr{D}$ (respectively, the minimal nonempty symmetric elements of $\mathscr{D}$ ).

Proof. Let the $R_{i}$ be the connected components of $R$ in the topology $\mathscr{D}$ for $R$ (respectively, in the topology $\mathscr{D}_{1}=\{S \in \mathscr{D} \mid S$ is symmetric\} for $R$ ).

DeFINITION 1.11. The open components (respectively the symmetric open components) of $R$ are the minimal nonempty elements of $\mathscr{D}$ (respectively $\mathscr{D}_{1}$ ).

CoROLlaRy 1.12. Let $\mathscr{D}$ be finite and express $R$ as the disjoint union $R=R_{1} \cup \cdots \cup R_{n}$ of its open (respectively symmetric open) components. Then a subset $S$ of $R$ is closed if and only if $S \cap S_{i}$ is closed for $1 \leqq i \leqq n$.

Proof. If $S$ is closed, then $S \cap R_{i}$ is closed since $R_{i}$ is closed for $1 \leqq i \leqq n$. Suppose, conversely, that $S \cap R_{i}$ is closed for $1 \leqq$ $i \leqq n$. Let $a, b \in S=S \cap R_{1} \cup \cdots \cup S \cap R_{n}$. If $a, b \in S \cap R_{i}$ for some $i$, then $\left\{a b, a^{-1} b, a b^{-1}\right\} \cap R \subset S \cap R_{i}$ since $S \cap R_{i}$ is closed $(1 \leqq i \leqq n)$. Thus, suppose that $a \in S \cap R_{i}, b \in S \cap R_{j}$ with $i \neq j$. Then $a \in R_{i}$ and $b \notin R_{i}$, so that $\left\{a b, a^{-1} b, a b^{-1}\right\} \cap R=\phi$ by Theorem 1.8 , since $R_{i}$ is open and closed. It follows that $\left(S^{2} \cup S^{-1} S \cup S S^{-1}\right) \cap R \subset S$ and $S$ is closed.

The above corollary determines the locology of $R$ in terms of the locology of its open components $R_{1}, \cdots, R_{n}$ for $\mathscr{D}$ finite.

CoRollary 1.13. For $\mathscr{D}$ finite, the set of connected components of $R$ (in the G-locology) is the union of the sects of connected components of the open (respectively symmetric open) components $R_{1}, \cdots, R_{n}$ of $R$. 
For the remainder of this section, we specialize to G-locological spaces $R$ where $R$ is the set of roots of a $G$-graded nonassociative algebra $A, G$ being a group. Here a nonassociative algebra is a vector space $A$ over a field $k$ and a product $x y \in A(x, y \in A)$ which is bilinear in the sense that

(1) $\quad(x+y) z=x z+y z(x, y, z \in A)$
(2) $x(y+z)=x y+x z(x, y, z \in A)$
(3) $\quad(c x) y=c(x y)=x(c y)(x, y \in A, c \in k)$

A subalgebra of $A$ is a subspace $B$ of $A$ such that $B^{2} \subset B$; and an ideal of $A$ is a subspace $B$ of $A$ such that $A B \subset B$ and $B A \subset B$. Here, $B C$ is the span of $\{b c \mid b \in B, c \in C\}$ and $B^{2}=B B . A$ G-graded nonassociative algebra, $G$ being a group, is a nonassociative algebra $A$ together with a $G$-grading of $A$, that is, a collection $\left\{A_{a} \mid a \in G\right\}$ of subspaces of $A$ indexed by $G$ such that

(1) $A=\sum_{a \in G} A_{a}$ (direct sum of subspaces);

(2) $A_{a} A_{b} \subset A_{a b}$ for all $a, b \in G$.

The set of roots of $A$ with respect to the $G$-grading of $A$ is $R=$ $\left\{a \in G \mid a \neq 1, A_{a} \neq 0\right\}$ where 1 is the identity of $G$ and 0 is the null space of $A$. The elements of $R$ are called roots. We let $H=A_{1}$, $A_{S}=\sum_{a \in S} A_{a}$ and $H_{S}=\sum_{a \in S} H_{a} H_{a^{-1}}+H_{a^{-1}} H_{a}$ for $S \subset R$.

We let $\langle B\rangle$ be the subalgebra of $A$ generated by $B$ for any subset $B$ of $A$.

Definition 1.14. We say that the G-graded nonassociative algebra $A$ is normal if

(1) for each $a \in G$ and $S \subset G, A_{a} A_{S}=0=A_{S} A_{a}$ implies that $A_{a}\left\langle A_{S}\right\rangle \subset\left\langle A_{S}\right\rangle$ and $\left\langle A_{S}\right\rangle A_{a} \subset\left\langle A_{S}\right\rangle$;

(2) $A_{1}\left\langle A_{S}\right\rangle \subset\left\langle A_{S}\right\rangle,\left\langle A_{S}\right\rangle A_{1} \subset\left\langle A_{S}\right\rangle$ for all $S \subset G$;

(3) $A_{1}\left(A_{a} A_{a^{-1}}\right) \subset A_{a} A_{a^{-1}}$ for all $a \in G$;

(4) $A_{S} B \subset B$ and $B A_{R} \subset B$ and $A_{S} \subset B$ imply that $\left\langle A_{S}\right\rangle \subset B$ for all $S \subset G$.

Note that graded Lie algebras and associative algeras are normal.

Theorem 1.15. Let $A$ be normal and let $S$ be a subset of $R$. Then

(1) for $S$ closed, $H_{S^{*}}$ is an ideal of $H$ and $\left\langle A_{S}\right\rangle=A_{S}+H_{S^{*}}$ where $S^{*}=S \cap S^{-1}$;

(2) for $S$ open and symmetric, $\left\langle A_{S}\right\rangle$ is an ideal of $A$ and $\left\langle A_{S}\right\rangle=A_{S}+A_{S}^{2}$

(3) for $S$ [open and closed, $\left\{R S \cup R S^{-1} \cup S R \cup S^{-1} R\right\} \cap R \subset S$, $\left\{S^{c} S \cup S^{c} S^{-1} \cup S S^{c} \cup S^{-1} S^{c}\right\} \cap R=\phi \quad$ and $\quad\left\{R S \cup R S^{-1} \cup S R \cup S^{-1} R\right\} \cap$ 
$\left\{R S^{c} \cup R\left(S^{c}\right)^{-1} \cup S^{c} R \cup\left(S^{c}\right)^{-1} R\right\} \cap R=\phi ;$

(4) for $S$ open, closed and symmetric, $\left\langle A_{S}\right\rangle=A_{S}+H_{S^{*}}$ is an ideal of $A$.

Proof. For (1), suppose that $S$ is closed. By normality, $H_{S^{*}}$ is an ideal of $A_{1}=H$. Clearly, $A_{S} H_{S^{*}} \cup H_{S^{*}} A_{S} \subset A_{S}$. Finally, $A_{S} A_{S} \subset$ $A_{S}+H_{S^{*}}$ since $S^{2} \cap R \subset S$. The first part of (3) follows from Theorem 1.7 for $S$ open and closed, since $\left(S^{2} \cup S S^{-1} \cup S^{-1} S\right) \cap R \subset S$; and the second and third parts follow from the first applied to both $S$ and $S^{c}$. Clearly, (4) follows from (1) and (2). For (2), assume that $S$ is open and symmetric and let $B=A_{S}+A_{S}^{2}$. Let $a \in S^{c}$. Since $S$ is symmetric, $a^{-1} \notin S$. By (3), $\left(S^{c} S \cup S S^{c}\right) \cap R=\phi$. Thus, $A_{a} A_{S}=0=A_{S} A_{a}$. By normality, therefore, $\left(A_{1}+A_{a}\right)\left\langle A_{S}\right\rangle \subset$ $\left\langle A_{S}\right\rangle$ and $\left\langle A_{S}\right\rangle\left(A_{1}+A_{a}\right) \subset\left\langle A_{S}\right\rangle$ for all $a \in S^{c}$. Thus, $\left\langle A_{S}\right\rangle$ is an ideal of $A$. It now remains only to show that $\left\langle A_{S}\right\rangle=B$, that is, that $B=A_{S}+A_{S}^{2}$ is a subalgebra of $A$. For this, it suffices, by normality, to show that $A_{S} B \cup B A_{S} \subset B$; for then $\left\langle A_{S}\right\rangle \subset B$ by normality, since $A_{S} \subset B$, so that $\left\langle A_{S}\right\rangle=B$. Since $B=A_{S}+A_{S}^{2}$, to show $A_{S} B \cup B A_{S} \subset B$ reduces to showing that $A_{S} A_{S}^{2} \cup A_{S}^{2} A_{S} \subset A_{S}+$ $A_{S}^{2}$. Therefore, consider $D=A_{a}\left(A_{b} A_{c}\right)$ where $a, b, c \in S$. If $a+b+$ $c \in S$ or $a+b+c \notin R$, then $D \subset B$. Thus, assume that $a+b+c \in S^{c}$. Since $S^{c}$ is closed, $a \notin S^{c}$, and $a=(a+b+c)-(b+c)$, we have $b+c \notin S^{c}$. But then either $b+c \in S$, in which case $D \subset A_{S}^{2}$; or $b+c \notin R$, in which case $D=A_{a}(0)=0$. Thus, in all cases, $D \subset B$. Thus, $A_{S} A_{S}^{2} \subset B$. Similarly, $A_{S}^{2} A_{S} \subset B$, and it follows that $\left\langle A_{S}\right\rangle \subset B$, therefore $\left\langle A_{S}\right\rangle=B$.

Definition 1.16. If $A^{2}=0, A$ is abelian. If $A$ has no ideals other than $A$ and $0, A$ is simple.

CoROLlaRy 1.17. For A simple and nonabelian and normal, $H_{S}=H$ for every nonempty symmetric open set $S$ of $R$.

Proof. By Theorem 1.15, $A_{S}+A_{S}^{2}$ must equal $A$, so that $H=$ $H_{S}$.

CoRollary 1.18. Let $A$ be normal and let $S, T$ be open and closed sets of $R$. Then $A_{S \cap T}+H_{(S \cap T)^{*}}$ and $\left\langle A_{S}\right\rangle \cap\left\langle A_{T}\right\rangle=A_{S \cap T}+$ $H_{S^{*}} \cap H_{T^{*}}$ are ideals of $A$.

Proof. This follows directly from Theorem 1.8 and 1.15 .

Some of our observations can now be summarized as follows. The proof is straight forward. 
THEOREM 1.19. Let $A$ be finite dimensional and normal, let $R_{1}, \cdots, R_{n}$ be the open components of $R$, let $A_{i}=A_{R_{i}}+H_{R_{i}^{*}}(1 \leqq i \leqq$ $n$ ) and let $I$ be the sum of all ideals of $A$ which are contained in $H$. Then

(1) the $A_{i}$ are ideals of $A(1 \leqq i \leqq n)$ and $A=H+A_{1}+$ $\cdots+A_{n}$

(2) $I$ is an ideal of $A$ contained in $H$ and $I A_{a}=0=A_{a} I$ for all $a \in R$;

(3) $\bar{A}=\bar{H} \oplus \bar{A}_{1} \oplus \cdots \oplus \bar{A}_{n}$ (direct sum) where $\bar{A}=A / I, \quad \bar{H}=$ $H+I / I$ and $\bar{A}_{i}=A_{i}+I / I(1 \leqq i \leqq n)$.

Finally, we specialize to the context of a finite dimensional Lie algebra $L$ over a field $k$ with split Cartan subalgebra $H$. Let $G$ be the group $G=F(H, k)$ with a product $a+b(a, b \in G)$ defined by $(a+b)(h)=a(h)+b(h)(h \in H)$. Then the Cartan decomposition $L=\sum_{a \in G} L_{a}$ is a $G$-grading for $L$ such that $H=L_{0}$. Let $R$ be the corresponding set of roots with the $G$-locology, so that $L=$ $H+\sum_{a \in R} L_{a}$.

Corollary 1.18 and Theorem 1.19 can now be refined as follows.

Corollary 1.20. Let $S, T \in \mathscr{D}$. Then

(1) $\left[L_{S}, L_{T}\right] \subset L_{S \cap T}+H_{S \cap T *}$ where $T_{*}=T \cup(-T)$;

(2) for $S$ and $T$ symmetric, $a \in S, b \in T$, we have $\left[L_{a}, L_{b}\right]=$ $\left[H_{a}, L_{b}\right]=\left[L_{a}, H_{b}\right]=\left[H_{a}, H_{b}\right]=0$ unless $a, b \in S \cap T$.

Proof. Since $(S+T) \cap R \subset(R+S) \cap(R+T) \cap R \subset S \cap T$ by Theorem 1.7, we have $\left[L_{S}, L_{T}\right] \subset L_{S \cap T}+H_{S \cap T *}$. Suppose next that $S$ and $T$ are symmetric, $a \in S$ and $b \in T$. If $a+b=0$ or $a-b=0$, then $a, b \in S \cap T$ by symmetry. Thus, suppose that $a+b \neq 0$ and $a-b \neq 0$. Then $a+b \notin R, a-b \notin R$ and $-a+b \notin R$ unless $a, b \in$ $S \cap T$, by Corollary 1.9. Since $\left[H_{a}, L_{b}\right]=\left[\left[L_{a}, L_{-a}\right], L_{b}\right]=\left[\left[L_{a}, L_{b}\right]\right.$, $\left.L_{-a}\right]+\left[L_{a},\left[L_{-a}, L_{b}\right]\right]$, it follows that $\left[H_{a}, L_{b}\right]=0$ unless $a, b \in S \cap T$ or $a,-b \in S \cap T$; that is, unless $a, b \in S \cap T$. And since $\left[H_{a}, H_{b}\right]=$ $\left[\left[H_{a}, L_{b}\right], L_{-b}\right]+\left[L_{b},\left[H_{a}, L_{-b}\right]\right]$, it follows that $\left[H_{a}, H_{b}\right]=0$ unless either $a, b \in S \cap T$ or $a,-b \in S \cap T$; that is unless $a, b \in S \cap T$.

CoROLlaRY 1.21. Let $R_{1}, \cdots, R_{n}$ be the symmetric open components of $R$ and let $L_{i}=L_{R_{i}}+H_{R_{i}}(1 \leqq i \leqq n)$. Then $L=H+L_{1}+$ $\cdots+L_{n},\left[L_{i}, L_{i}\right] \subset L_{i},\left[L_{i}, L_{j}\right]=0$ for $1 \leqq i, j \leqq n$ and $i \neq j$ and $L^{\infty}=L_{1}+\cdots+L_{n}$.

Proof. Since $R=R_{1} \cup \cdots \cup R_{n}$ (disjoint union of symmetric 
open and closed sets), this follows directly from Corollary 1.20 and the fact proved in Winter [4] that $L^{\infty}=\sum_{a \in R}\left[L_{a}, L_{-a}\right]+\sum_{a \in R} L_{a}$.

Before turning to the next section, we mention that the set $\mathscr{D}$ of open and closed (respectively symmetric open and closed) sets of a $G$-locology for $R$ determine a topology $\langle\mathscr{D}\rangle$ for $R$ as defined below. Our use of this topology has been restricted to the case where $\mathscr{D}$ is finite, in which case $\mathscr{D}=\langle\mathscr{D}\rangle$. That $\langle\mathscr{D}\rangle$ is, in general, a topology for $R$ is evident.

Definition 1.22. The set $\langle\mathscr{D}\rangle$ of unions of subsets of $\mathscr{D}$ is called the G-topology (respectively symmetric G-topology) for $R$.

2. Idempotent nonassociative algebras and Lie algebras. In this section, all nonassociative algebras are finite dimensional.

Definition 2.1. In a nonassociative algebra $A$, an idempotent is a subalgebra $E$ of $A$ such that $E=E^{2} \neq 0$. If $E \supseteqq E_{1}, E_{1}$ is proper in $E$. If $E_{1} E_{2}=0=E_{2} E_{1}, E_{1}$ and $E_{2}$ are orthogonal. If an idempotent $E$ cannot be written as $E=E_{1}+E_{2}$ where $E_{1}$ and $E_{2}$ are proper orthogonol idempotents in $E$, then $E$ is a primitive idempotent. The identity of $A$ is $1_{A}=A^{(\infty)}=\bigcap_{i=1}^{\infty} A^{(i)}$; where $A^{(1)}=$ $A^{2}$ and $A^{(i+1)}=A^{(i) 2}$ for all $i$. An idempotent $E$ of $A$ is central if either $1_{A}=E$ or $1_{A}=E+F$ where $E$ and $F$ are orthogonol idempotents. If $A=A^{2} \neq 0, A$ is an idempotent algebra. And $A$ is primitive if $A$ is a primitive idempotent of $A$.

Note that $1_{A}=0$ if and only if $A$ is solvable in the sense that $A^{(i)}=0$ for some $i$. For $A$ nonsolvable, $1_{A}$ is an idempotent of $A$ and $1_{A}$ contains every idempotent $E$ of $A$. If $A=A^{2} \neq 0$, then $A=1_{A}$, in which case $A$ is an idempotent algebra. If $E$ is a central idempotent of $A$, we have $1_{A} E=E 1_{A}=E$, since $1_{A}=E+F$ where $(E+F) E=E(E+F)=E$.

It is possible to align our language even more closely with the classical theory of idempotents by noting that each central idempotent $E$ of $A$ determines a unique minimal central idempotent, called $1_{A}-E$, such that $1_{A}-E$ and $E$ are orthogonal and such that $1_{A}=E+\left(1_{A}-E\right)$. For if $1_{A}=E+F=E+G$ where $F$ and $G$ are central idempotents orthogonal to $A$, then $1_{A}=L_{A}^{2}=E+F G=$ $E+F \cap G=E+(F \cap G)^{(\infty)}=E+H$ where $H$ is the central idempotent $(F \cap G)^{(\infty)}$ contained in $F \cap G$.

TheOREM 2.2. A nonassociative algebra $A$ has only finitely many central primitive idempotents $E_{1}, \cdots, E_{n}$. They are pairwise orthogonol and their sum is $1_{A}=E_{1}+\cdots+E_{n}$. Every central 
idempotent $E$ of $A$ is the sum $E=\sum_{E E_{i} \neq 0} E_{i}$ of those $E_{i}$ not orthogonol to $E$. In particular, $A$ has only finitely many central idempotents.

Proof. We claim first that any central idempotent $E$ of $A$ can be written as $E=E_{1}+\cdots+E_{m}$ where the $E_{i}$ are pairwise orthogonol central primitive idempotents. We use induction on the dimension of $E$. If $E$ is primitive (as when $E$ has dimension 1), we take $E=E_{1}$. Otherwise, we can write $E=F+G$ where $F$ and $G$ are proper orthogonol idempotents. Since $E$ is central, so are $F$ and $G$. By induction, we may write both $F$ and $G$, and therefore also $E$, as sum $E=E_{1}+\cdots+E_{m}$ of pairwise orthogonol central primitive idempotents, as claimed. Since either $1_{A}=E$ or $1_{A}=E+F$ where $[E, F]=0$ and $F$ is a central idempotent, we can write $F=$ $E_{m+1}+\cdots+E_{n}$ and $1_{A}=E_{1}+\cdots+E_{n}$ where the $E_{i}$ are pairwise orthogonal central primitive idempotents for $1 \leqq i \leqq n$. Let $P$ be any central primitive idempotent. Then $P=1_{A} P=P 1_{A}=P E_{1}+$ $\cdots+P E_{n}=E_{1} P+\cdots+E_{n} P$ and $P E_{i} \cup E_{i} P \subset P \cap E_{i}$ for all $i$. Thus, $P E_{i} \neq 0$ for some $i$, say $i=1$, without loss of generality. We claim that $P=E_{1}$, since $P E_{1} \neq 0$. We have $P=P^{(\infty)}=P_{1}+\cdots+P_{n}$ where $P_{j}=\left(P \cap E_{j}\right)^{(\infty)}$. Since $P_{i}^{2}=P_{i}$ and $P_{j} P_{i}=0=P_{i} P_{j}$ for $i \neq j$, $P=P_{j}$ for some $j$. Thus, $P \subset E_{j}$. Since $P E_{1} \neq 0$, we have $j=1$ and $P \subset E_{1}$. If $P=1_{A}$, then $1_{A}=P=E_{1}$, and we are done. Otherwise, write $1_{A}=P+Q$ where $P$ and $Q$ are orthogonol central idempotents. Then $E_{1}=E_{1} 1_{A}=E_{1} P+E_{1} Q=P+E_{1} \cap Q=P+P^{\prime}$ where $P^{\prime}=\left(E_{1} \cap Q\right)^{(\infty)}$. Thus, $P^{\prime}=0$ and $E_{1}=P$; for otherwise $P^{\prime}$ is an idempotent orthogonol to $P$ and $E_{1}$ is not primitive.

THEOREM 2.3. Let $G$ be the connected component of the identity of the automorphism group Aut $A$ of a nonassociative algebra $A$. Then $G$ and its Lie algebra $\dot{G}$ stabilize each central idempotent of A. If the characteristic is 0 , the central idempotents are stable under the derivations of $A$. And if $A$ is a Lie algebra of characteristic 0 , the central idempotents are ideals of $A$.

Proof. The subgroup $H$ of elements of $G$ which stabilize each central idempotent of $A$ is closed. Furthermore, $G$ permutes the central idempotents of $A$. Since there are only finitely many, by Theorem 2.2, $G: H$ is finite. But then $H$ is open, since $H$ and its finitely many cosets are closed. Thus, $H$ is open and closed, so that $G=H$ by the connectedness of $G$. Thus, the central idempotents of $A$ are stable under $G$, therefore under $\dot{G}$. In characteristic $0, \dot{G}=\operatorname{Der} A$, where $\operatorname{Der} A$ is the algebra of derivations of $A$. If $A$ is a Lie algebra of characteristic 0 , we therefore have ad $A \subset$ 
$\operatorname{Der} A \subset \dot{G}$, so that the central idempotents of $A$ are ad $A$-stable, that is, they are ideals of $A$.

CoROLlaRY 2.4. Let the central idempotents of $A$ be $E_{1}, \cdots, E_{n}$. Then for any idempotent ideal $I$ of $1_{A}, I=I_{1}+\cdots+I_{n}$ where $I_{i}$ is an idempotent of $E_{i}(1 \leqq i \leqq n)$. If $A$ is a Lie algebra, these $I_{i}$ can be taken to be ideals of $1_{A}$.

Proof. $I=1_{A} I=\sum_{i=1}^{n} E_{i} I \subset \sum_{i=1}^{n} E_{i} \cap I \subset I$ and $I=\sum_{i=1}^{n} I_{i}$ where $I_{i}=\left(E_{i} \cap I\right)^{(\infty)}$. Note that $I_{i}$ is an ideals of $1_{A}$ if $A$ is a Lie algebra.

Corollary 2.5. Suppose that $L$ is a Lie algebra. Then the Cartan subalgebras $H$ of $1_{L}=L^{(\infty)}$ are the subalgebras $H=H_{1}+$ $\cdots+H_{n}$ where the central primitive idempotents are $E_{1}, \cdots, E_{n}$ are $H_{i}$ is a Cartan subalgebra of $E_{i}$ for $1 \leqq i \leqq n$. For each such $H, H_{i}=E_{i} \cap H$ for $1 \leqq i \leqq n$.

Proof. Each such $H$ is a Cartan subalgebra of $1_{L}$, since $\left(1_{L}\right)_{0}(\operatorname{ad} H)=\sum_{i=1}^{n}\left(E_{i}\right)_{0}(\operatorname{ad} H)=\sum_{i=1}^{n}\left(E_{i}\right)_{0}\left(\operatorname{ad} H_{i}\right)=\sum_{i=1}^{n} H_{i}=H$. Conversly, let $H$ be a Cartan subalgebra of $1_{L}=L^{(\infty)}$. Let $H_{i}=E_{i} \cap$ $\left(H+\sum_{i \neq j} E_{j}\right)$ for $1 \leqq i \leqq n$, and note that $H \subset H_{1}+\cdots+H_{n}$ since $H \subset E_{1}+\cdots+E_{n}$. We may conclude that $H_{i} \subset\left(E_{i}\right)_{0}\left(\operatorname{ad} H_{i}\right) \subset$ $\left(E_{i}\right)_{0}($ ad $H)=E_{i} \cap H \subset H$ for $1 \leqq i \leqq n$, so that $H=H_{1}+\cdots+H_{n}$. But then $H_{i}=E_{i} \cap H=\left(E_{i}\right)_{0}(\operatorname{ad} H)=\left(E_{i}\right)_{0}\left(\operatorname{ad} H_{i}\right)$ and $H_{i}$ is a Cartan subalgebra of $E_{i}$ for $1 \leqq i \leqq n$.

Note that the Cartan subalgebra $H$, in the above theorem, is split if and only if $H_{i}$ is split for $1 \leqq i \leqq n$. In the proofs of Theorems 2.6 and 3.3, we make use of $\left[H_{i}, H_{j}\right]=0$ for $i \neq j$ to conclude that $R\left(X_{i} \cup X_{j}\right)=R\left(H_{1} \cup \cdots \cup H_{n}\right)=R\left(H_{1}+\cdots+H_{n}\right)=R(H)$.

THEOREM 2.6. Let $H$ be a split Cartan subalgebra of an idempotent Lie algebra $L$, and let $R=R_{1} \cup \cdots \cup R_{n}$ be the decomposition of the set $R$ of roots of $H$ into its connected components $R_{i}(1 \leqq i \leqq n)$ in the symmetric $G$-locology for $R$ where $G=F(H, k)$. Then

(1) $R_{i}$ is open and closed for $1 \leqq i \leqq n$;

(2) the ideals $E_{i}=\left\langle L_{R_{i}}\right\rangle=L_{R_{i}}+H_{R_{i}}(1 \leqq i \leqq n)$ are the central primitive idempotents of $L$ so that $L=E_{1}+\cdots+E_{n}$, $\left[E_{i}, E_{j}\right]=0$ for $i \neq j$;

(3) $L$ is primitive if and only if $R$ is connected.

Proof. Let $E_{1}, \cdots, E_{m}$ be the central primitive idempotents of 
$L$ and $H_{i}=H \cap E_{i}(1 \leqq i \leqq m)$. By Theorem 2.2 and Corollary 2.5, $L=E_{1}+\cdots+E_{m}, H_{i}$ is a split Cartan subalgebra of $E_{i}(1 \leqq i \leqq m)$ and $H=H_{1}+\cdots+H_{m}$. Let $X_{i}=\bigcup_{j=1}^{m} H_{j}-H_{i}$ and $R_{i}=R\left(x_{i}\right)$ $(1 \leqq i \leqq m)$. We claim that the $R_{i}$, which are closed, are also open; and that the $R_{i}$ are, in fact, the connected components of $R$. Note first that $R_{i} \cap R_{j}=R\left(X_{i} \cup X_{j}\right)=R(H)=\phi$ for $i \neq j$. Next, let $a \in R$, so that $0 \neq L_{a}(\operatorname{ad} H)=\sum\left(E_{i}\right)_{a}\left(\operatorname{ad} H_{i}\right)$ and $0 \neq\left(E_{i}\right)_{a}\left(\operatorname{ad} H_{i}\right)$ for some $i$. Then $0=\left(E_{i}\right)_{a}\left(\operatorname{ad} H_{j}\right)$ since $\left[E_{i}, E_{j}\right]=0$, so that $a\left(H_{j}\right)=0$ for $i \neq j$. Thus, $a \in R\left(X_{i}\right)=R_{i}$. It follows that $R=R_{1} \cup \cdots \cup R_{m}$ (disjoint union of closed sets). Furthermore, $a\left(H_{i}\right) \neq 0$, and we see easily that $R_{i}$ therefore is also $R_{i}=R-R\left(H_{i}\right)$, an open set $(1 \leqq i \leqq m)$. Moreover, we see that $R_{i}=\left\{a \in R \mid\left(L_{i}\right)_{a}(\operatorname{ad} H) \neq\{0\}\right\}$ $(1 \leqq i \leqq m)$. Since $R_{i} \cap R_{j}=\phi$ for $i \neq j$, it follows that $E_{i}$ contains $L_{R_{i}}$ and $E_{i} \cap L_{R_{j}}=0$ for $1 \leqq i, j \leqq m$ and $i \neq j$. Since $R_{i}$ is open, closed and symmetric, $F_{i}=L_{R_{i}}+H_{R_{i}}$ is an ideal of $L(1 \leqq i \leqq m)$. Since $E_{i} \supset\left\langle L_{R_{i}}\right\rangle=F_{i}$, since $F_{i}^{2}=F_{i}(1 \leqq i \leqq m)$ and since $L=L^{2}=$ $L_{R}+H_{R}=F_{1}+\cdots+F_{m}$, the $F_{i}$ are central idempotents of $L$. It follows easily from Theorem 2.2 that $E_{i}=F_{i}$, so that $E_{i}=L_{R_{i}}+H_{R_{i}}$ $(1 \leqq i \leqq m)$. For (1) and (2), it now remains only to show that $R_{i}$ is connected. Thus, suppose that $R_{i}=S \cup T$ (disjoint union) where $S, T$ are relatively closed and symmetric in $R_{i}$. Since $S$ and $T$ are relatively closed and symmetric in $R_{i}$, and disjoint, $S$ and $T$ are relatively open in $R_{i}$. It follows that, in the Lie algebra $L_{i}=L_{R_{i}}+$ $H, S$ and $T$ are open, closed and symmetric. Thus, $\left[L_{S}, L_{T}\right]=0$ by Corollary 1.2, since $S \cap T=\phi$. It follows that $E_{i}=L_{R_{i}}+H_{R_{i}}=E+F$ where $E=L_{S}+H_{S}, F=L_{T}+H_{T}, E^{2}=E, F^{2}=F, E F=0$. Since $E_{i}$ is primitive, $E_{i}=E$ or $F_{i}=F$ and $T=\phi$ or $S=\phi$. It follows that $R_{i}$ is connected $(1 \leqq i \leqq m)$. In particular $m=n$. Now (3) follows from (1) and (2), and all assertions have been established.

CoRollary 2.7. For a Lie algebra $L$ with split Cartan subalgebra $H$ and set $R$ of roots, if $L$ is semisimple (characteristic 0 ) or classical (characteristic $p>0$ ), then the connected components $R_{i}$ of $R$ in the symmetric G-locology are the irreducible root systems of $R$ in the sense of Bourbaki [1].

In the proof of Theorem 2.6, it is actually shown that the $R_{i}$ are open and closed in the locology $\{R(x) \mid X \subset H\}$ which, a priori, is a coarser locology than the symmetric G-locology. On the other hand, the $R_{i}$ are also the connected components of $R$ in the symmetric $G$-topology of $R$.

3. Ideal structure and locology of a Lie algebra and its root spaces. In this section, we consider a finite dimensional Lie algebra 
$L$ with split Cartan subalgebra $H$ and corresponding set $R$ of roots with the symmetric $G$-locology of 1.2, 1.20.

THEOREM 3.1. Let $L=L_{1}+\cdots+L_{n}$ (sum of ideals) where $\left[L_{i}, L_{j}\right]=0$ for $1 \leqq i, j \leqq n$ and $i \neq j$. Then

(1) $H=H_{1}+\cdots+H_{n}$ and $R=R_{1} \cup \cdots \cup R_{n}$ (disjoint) where $H_{i}=H \cap L_{i}$ and $R_{i}=\left\{a \in R \mid\left(L_{i}\right)_{a}(\operatorname{ad} H) \neq 0\right\}$ for $1 \leqq i \leqq n$;

(2) $R_{i}$ is open and closed, $H_{i}$ is a Cartan subalgebra of $L_{i}$ and $L_{i}=H_{i}+L_{R_{i}}$ for $1 \leqq i \leqq n$;

(3) $L^{\infty}=\sum L_{i}^{\infty}, L_{i}^{\infty}=L_{R_{i}}+H_{R_{i}}$ and $\left[L, L_{i}^{\infty}\right]=L_{i}^{\infty}$ for $1 \leqq i \leqq n$.

Proof. As in the proof of Theorem 2.6, we see that $H=H_{1}+$ $\cdots+H_{n}, R=R_{1} \cup \cdots \cup R_{n}$ (disjoint), $R_{i}$ is open and closed and $H_{i}$ is a Cartan subalgebra of $L_{i}$ for $1 \leqq i \leqq n$. For $a \in R_{i}$, we have $a \notin R_{j}$ and therefore $\left(L_{j}\right)_{a}(\operatorname{ad} H)=0$ for $i \neq j$. It follows that the decomposition of $L_{i}$ under ad $H$ is $L_{i}=H_{i}+\sum_{a \in R_{i}} L_{a}=H_{i}+L_{R_{i}}$. Clearly $L^{\infty}=L_{1}^{\infty}+\cdots+L_{n}^{\infty}$, since $\left[L_{i}, L_{j}\right]=0$ for $i \neq j$. Since $L_{i} \supset$ $L_{R_{i}}$ and $\left[L, L_{i}^{m}\right]=L_{i}^{m+1}$ for all $m$, we have $L_{i} \supset L_{R_{i}}, L_{i}^{2}=\left[L, L_{i}\right] \supset$ $L_{R_{i}}, \cdots$. Thus $L_{i}^{\infty} \supset L_{R_{i}}$. Since $L_{R_{i}}+H_{R_{i}}$ is and ideal of $L_{i}$ and $L_{i} /\left(L_{R_{i}}+H_{R_{i}}\right)$ is nilpotent, we also have $L_{i} \subset L_{R_{i}}+H_{R_{i}}$, so that $L_{i}=L_{R_{i}}+H_{R_{i}}$ for $1 \leqq i \leqq n$. That $\left[L, L_{i}^{\infty}\right]=L_{i}^{\infty}$ is clear since $L=$ $L_{1}+\cdots+L_{n}$ and $\left[L_{i}, L_{j}\right]=0$ for $i \neq j$.

The following theorem is proved in Winter [3] and, under a stronger hypothesis, in Winter [2].

THEOREM 3.2. Let $L$ be a Lie algebra, $I$ and ideal of $L$. Suppose that either the characteristic $p$ of $L$ is 0 or $\left(\operatorname{ad}_{I} I\right)^{p} \subset \operatorname{ad}_{I} I$. Then $I_{0}(\operatorname{ad}(H \cap I))$ is a Cartan subalgebra of $I$ for every Cartan subalgebra $H$ of $L$.

THEOREM 3.3. Let $I$ be an ideal of $L$ and suppose that $I_{0}(\operatorname{ad} H \cap I)$ is a Cartan subalgebra of $I$. Let $I=I_{1}+\cdots+I_{n}$ (sum of ideals) where $\left[I_{i}, I_{j}\right]=0$ for $1 \leqq i, j \leqq n$ and $i \neq j$. Then

(1) $H_{I}=H_{1}+\cdots+H_{n}$ and $R_{i}=R_{1} \cup \cdots \cup R_{n} \cup S$ (disjoint) where $H_{I}=H \cap I, \quad R_{i}=\left\{a \in R \mid I_{a}\left(H_{I}\right) \neq 0\right\}, \quad S=R_{I}\left(H_{I}\right)$ and $R_{i}=$ $\left\{a \in R-S \mid I_{i a}\left(H_{I}\right) \neq 0\right\}$ for $1 \leqq i \leqq n$;

(2) $R_{i}$ is relatively open and closed in $R_{I}-S, H_{i}+I_{i S}$ is a Cartan subalgebra of $I_{i}$ and $I_{i}=\left(H_{i}+I_{i S}\right)+I_{R_{i}}$ for $1 \leqq i \leqq n$.

Proof. $I_{0}\left(\right.$ ad $\left.I_{H}\right)=H_{I}+I_{S}$ is a Cartan subalgebra of $I$ by Theorem 3.2. We have $H_{I}=I_{0}(\operatorname{ad} H)=\sum_{i=1}^{n} I_{i 0}(\operatorname{ad} H)=\sum_{i=1}^{n} H_{i}$. Letting $X_{i}=\mathrm{U}_{j=1}^{n} H_{j}-H_{i}$ and $\hat{R}_{i}=R_{I}\left(X_{i}\right)$ for $1 \leqq i \leqq n$, we have $\hat{R}_{i} \cap \hat{R}_{j}=R_{I}\left(X_{i} \cup X_{j}\right)=R\left(H_{1} \cup \cdots \cup H_{n}\right)=R\left(H_{1}+\cdots+H_{n}\right)=R_{I}\left(H_{I}\right)=S$ 
for all $i \neq j$. Here, we use the fact that $\left[h_{i}, h_{j}\right]=0\left(h_{i} \in H_{i}\right)$ for all $i \neq j$ implies that $a\left(h_{1}+\cdots+h_{n}\right)=a\left(h_{1}\right)+\cdots+a\left(h_{n}\right)$. Let $R_{i}$ be the complement of $S$ in $\widehat{R_{i}}$, so that $R_{i} \cap R_{j}=\phi$ for $i \neq j$. For $a \in$ $R_{I}-S$, we have $0 \neq I_{i a}\left(H_{I}\right)=I_{i a}\left(\operatorname{ad} H_{i}\right)$ for some $i$; and therefore $a\left(H_{j}\right)=0$ for $j \neq i$; and therefore $a\left(H_{i}\right) \neq 0$ and $a \in \widehat{R_{i}}-S=R_{i}$. It follows that $R_{I}=R_{1} \cup \cdots \cup R_{n} \cup S$ (disjoint), with $R_{i}$ relatively open and closed in $R_{I}-S$. It also follows that $I_{i}=I_{i 0}\left(\operatorname{ad} H_{I}\right)+$ $\sum_{a \in R_{i}} I_{a}=\left(H_{i}+I_{i S}\right)+I_{R_{i}}$. As in the proof of Theorem 3.1, $K=$ $H_{I}+I_{S}$ is Cartan subalgebra of $I$ implies that $K_{i}=K \cap I_{i}=H_{i}+I_{i S}$ is a Cartan subalgebra of $I_{i}$ for $1 \leqq i \leqq n$.

We can now improve Corollary 1.21 and use it and Theorem 3.3 to prove that if $H_{\infty}=H \cap L^{\infty}$ is a Cartan subalgebra of $L$, the connected components $R_{i}$ of $R$ in the symmetric $G$-locology are both open and closed. Whether this is true when $H_{\infty}$ is not a Cartan subalgebra of $L^{\infty}$ is an open question, the answer of which is probably negative.

THEOREM 3.4. Let $R_{1}, \cdots, R_{n}$ be the connected components of $R$, in the symmetric G-locology, and let $L_{i}=L_{R_{i}}+H_{R_{i}}(1 \leqq i \leqq n)$. Then $\left[L_{i}, L_{i}\right] \subset L_{i},\left[L_{i}, L_{j}\right]=0$ for $i \neq j$ and $L^{\infty}=L_{1}+\cdots+L_{n}$.

Proof. Choose a decomposition $R=R_{1} \cup \cdots \cup R_{n}$ (disjoint) with $n$ maximal satisfying all of the following conditions:

(1) The $R_{i}$ are closed, nonempty, pairwise disjoint;

(2) every connected subset of $R$ is contained in some $R_{i}$;

(3) the conclusion of the Theorem 3.4 holds.

We claim that the $R_{i}$ are the connected components of $R$, that is, that each $R_{i}$ is connected. If $R_{n}$ is not connected, then $R_{n}=R_{n}^{\prime} \cup$ $R_{n+1}^{\prime}$ (nonempty, closed, disjoint) and each connected subset of $R_{n}$ is either in $R_{n}^{\prime}$ or in $R_{n+1}^{\prime}$. In the context of the Lie algebra $L_{n}=$ $L_{R_{n}}+H_{R_{n}}, R_{n}^{\prime}$ and $R_{n+1}^{\prime}$ are relatively closed and open, so that $L_{n}=$ $L_{a}+L_{b}$ with $L_{a}^{2} \subset L_{a}, L_{b}^{2} \subset L_{b},\left[L_{a}, L_{b}\right]=0$ where $L_{a}=L_{R_{n}^{\prime}}+H_{R_{n}^{\prime}}$ and $L_{b}=L_{R_{n+1}^{\prime}}+H_{R_{n+1}^{\prime}}$. Thus, $R_{1}, \cdots, R_{n-1}, R_{n}^{\prime}, R_{n+1}^{\prime}$ satisfies conditions (1), (2), (3), a contradition. We must conclude that $R_{n}$ (and, similarly, $R_{i}$ for all $i$ ) is connected as asserted. Note that the assertion $L^{\infty}=L_{1}+\cdots+L_{n}$ is verified as in Corollary 1.21.

CoRollary 3.5. Suppose that $H_{\infty}=H \cap L^{\infty}$ is a Cartan subalgebra of $L^{\infty}$. Then

(1) the connected components $R_{i}(1 \leqq i \leqq n)$ of $R$ are both open and closed;

(2) $H_{R_{i}}$ is a Cartan subalgebra of $L_{R_{i}}+H_{R_{i}}=L_{i}(1 \leqq i \leqq n)$ 
and $L^{\infty}=L_{1}+\cdots+L_{n}$ (sum of ideals of $\left.L\right)$ where $\left[L_{i}, L_{j}\right]=0$ for $i \neq j$.

Proof. We have (2) by Theorem 3.4 and the hypothesis. Thus, by Theorem 3.3, $R_{i}$ is open and closed in $R_{I}-S=R-S=R-$ $R\left(H_{\infty}\right)=R-\phi=R$.

Finally, we note that Theorem 3.4 is in the direction of a converse to Theorem 3.1. It provides a decomposition $L^{\infty}=L_{1}+\cdots+L_{n}$ where $L_{i}=L_{R_{i}}+H_{R_{i}}$ and the $R_{i}$ are the connected components of $R$. It follows immediately that the same is true if the $R_{1}, \cdots, R_{n}$ are pairwise disjoint and every connected component of $R$ is contained in one of the $R_{i}$ as is the case when $R=R_{1} \cup \cdots \cup R_{n}$ is disjoint union of open and closed sets (the situation which immerges in Theorem 3.1). Although it may not be possible to lift such a decomposition $L^{\infty}=L_{1}+\cdots+L_{n}$ to a decomposition $L=\bar{L}_{1}+\cdots \bar{L}_{n}$ of $L$ (compare with the hypothesis of Theorem 3.1), the following lifting is possible when $H$ is abelian.

THEOREM 3.6. Let $H$ be abelian and let $L^{\infty}=L_{1}+\cdots+L_{n}$ with $L_{i}=L_{R_{i}}+H_{R_{i}}, \quad R=R_{1} \cup \cdots \cup R_{n}$ (disjoint) and $\left[L_{i}, L_{i}\right] \subset L_{i}$, $\left[L_{i}, L_{j}\right]=0$ for all $i \neq j$. Then there is a Lie algebra $\hat{L}$ containing $L$ as ideal and decomposition $\hat{L}=\hat{L}_{1}+\cdots+\hat{L}_{n}$ (sum of ideals such that $\left[\hat{L}_{i}, \hat{L}_{j}\right]=0$ for $i \neq j$ and $\hat{L}_{i} \cap L=L_{i}(1 \leqq i \leqq n)$.

Proof. $L$ is ideal of $M=(\operatorname{Der} L) \oplus L$ (semidirect) where $[D, x]=$ $D(x)$ for $D \in \operatorname{Der} L, x \in L$. Let $h \in H$ and define $D_{i}: L \rightarrow L$ so that $D_{i}$ is linear, $D_{i}(H)=0,\left.D_{i}\right|_{L_{R_{i}}}=\left.\operatorname{ad} h\right|_{L_{R_{i}}} . \quad D_{i}\left(L_{R_{i}}\right)=0$ for $i \neq j$. One easily verifies that $D_{i} \in \operatorname{Der} L(1 \leqq i \leqq n)$. Since $D_{i}$ depends on $h$, we use the notation $D_{i}=D_{i}(h)$. The span $\hat{H}_{0}$ of $\left\{D_{i}(h) \mid 1 \leqq i \leqq n\right.$, $h \in H\}$ is a commutative subalgebra of Der $L$ and we let $\hat{L}=\hat{H}_{0}+L$ and $\hat{H}=\hat{H}_{0}+H$. Clearly $\hat{H}$ is a Cartan subalgebra of $\hat{L}$. Let $\hat{H}_{i}=\left\{x \in \hat{L} \mid\left[x, L_{j}\right]=0\right.$ for all $i \neq j$ and $\left.[x, H]=0\right\}$. We claim that $\hat{H}=\hat{H}_{1}+\cdots+\hat{H}_{n}$. Clearly, $\hat{H}_{1}+\cdots+\hat{H}_{n}$ contains $\hat{H}_{0}$. Let $h \in H$ and $x=h-\left(D_{1}(h)+\cdots+D_{n}(h)\right)$. Then $\left[x, L_{i}\right]=0$ for $1 \leqq i \leqq n$. Furthermore, $\left[x, H_{0}\right]=0$. Finally, $\left[x, \hat{H}_{0}\right]=0$. It follows that $x$ centralizes $\hat{L}$. In particular, $x \in \hat{L}_{0}(\operatorname{ad} \hat{H})=\hat{H}$. It follows that $x \in$ $\hat{H}_{i}$ for all $i$ and that $h=x+D_{1}(h)+\cdots+D_{n}(h) \in \hat{H}_{1}+\cdots+\hat{H}_{n}$. Thus, $H \subset \hat{H}_{1}+\cdots+\hat{H}_{n}$, so that $\hat{H} \subset \hat{H}_{1}+\cdots+\hat{H}_{n}$. Since $\left[\hat{H}_{1}, H\right]=0$ and $\left[\hat{H}_{i}, L_{j}\right]=0$ for $i \neq j$, we have $\left[\hat{H}_{i}, D_{i}(H)\right]=0$ and $\left[\hat{H}_{i}, D_{j}(H)\right]=0$ for $i \neq j$, so that $\left[\hat{H}_{i}, \hat{H}_{0}\right]=0$. It follows that $\hat{H}_{i} \subset \hat{L}_{0}(\operatorname{ad} \hat{H})=\hat{H}$ $(1 \leqq i \leqq n)$. Thus, $\hat{H}=\hat{H}_{1}+\cdots+\hat{H}_{n}$. Let $\hat{L}_{i}=\hat{H}_{i}+L_{i}(1 \leqq i \leqq n)$. It is then evident that $\hat{L}=\hat{L}_{1}+\cdots+\hat{L}_{n}$ is a decomposition satisfying the asserted conditions. 
Clearly, the $R_{i}$ in Theorem 3.6 are open and closed in $R$ in the locology defined by $\hat{H}$.

\section{REFERENCES}

1. N. Bourbaki, Groupes et Algèbres de Lie, Paris, Hermann, 1960.

2. David J. Winter, Cartan subalgebras of a Lie algebra and its ideals, Pacific J. Math., 33, No. 2, (1970), 537-541.

3. - Cartan subalgebras of a Lie algebra and its ideals II, (in preparation).

4. —_ Cartan decompositions and Engel subalgebra triangulability, J. Algebra, 62 (to appear).

Received March 10, 1980. This reserch was supported in part by the National Science Foundation.

UNIVERSITY OF MichigaN

ANN ARBOR, MI 48109 


\section{PACIFIC JOURNAL OF MATHEMATICS}

\section{EDITORS}

DONALD BABBITT (Managing Editor)

University of California

Los Angeles, California 90024

\section{Hugo Rossi}

University of Utah

Salt Lake City, UT 84112

C. C. MOore and Arthur AGuS

University of California

Berkeley, CA 94720
J. DugundJI

Department of Mathematics University of Southern California Los Angeles, California 90007

R. FinN and J. Milgram Stanford University Stanford, California 94305

ASSOCIATE EDITORS
R. ARENS
E. F. BECKENBACH
B. H. NeumanN
F. WOLF
K. YOSHIDA

\section{SUPPORTING INSTITUTIONS}

UNIVERSITY OF ARIZONA

UNIVERSITY OF BRITISH COLUMBIA

CALIFORNIA INSTITUTE OF TECHNOLOGY

UNIVERSITY OF CALIFORNIA

MONTANA STATE UNIVERSITY

UNIVERSITY OF NEVADA, RENO

NEW MEXICO STATE UNIVERSITY

OREGON STATE UNIVERSITY
UNIVERSITY OF OREGON

UNIVERSITY OF SOUTHERN CALIFORNIA

STANFORD UNIVERSITY

UNIVERSITY OF HAWAII

UNIVERSITY OF TOKYO

UNIVERSITY OF UTAH

WASHINGTON STATE UNIVERSITY

UNIVERSITY OF WASHINGTON 


\section{Pacific Journal of Mathematics}

Vol. 99, No. $1 \quad$ May, 1982

Mariano Giaquinta, Jindrich Necas, O. John and J. Stará, On the

regularity up to the boundary for second order nonlinear elliptic systems . . 1

Siegfried Graf, Realizing automorphisms of quotients of product $\sigma$-fields . . 19

Alfred Washington Hales and Ernst Gabor Straus, Projective colorings . . . 31

Sandra Hayes, The weak Nullstellensatz for finite-dimensional complex

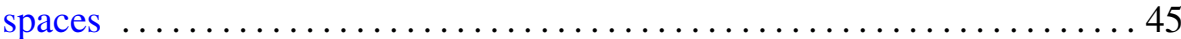

Gerald Norman Hile and Murray Harold Protter, The Cauchy problem

and asymptotic decay for solutions of differential inequalities in Hilbert

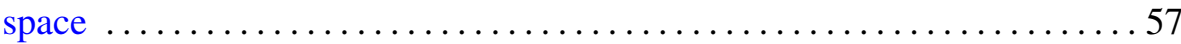

Robert D. Little, Projective space as a branched covering with orientable

branch set ......................................... 89

Jaroslav Mach, On the proximinality of Stone-Weierstrass subspaces . . . . . 997

John C. Morgan, II, On product bases ...................... 105

K. Balakrishna Reddy and P. V. Subrahmanyam, Altman's contractors

and fixed points of multivalued mappings . .................. 127

James Ted Rogers Jr., Decompositions of homogeneous continua . . . . . . . 137

Ahmed Ramzy Sourour, Characterization and order properties of

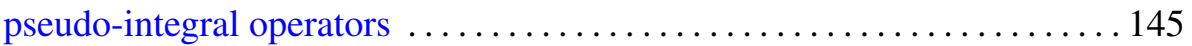

Robert Moffatt Stephenson Jr., Pseudocompact and Stone-Weierstrass

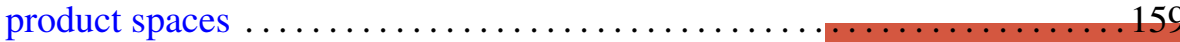

Bruce Stewart Trace, On attaching 3-handles to a 1-connected

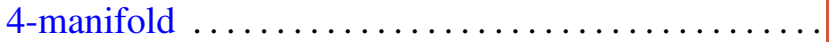

Akihito Uchiyama, The construction of certain BMO functions and the corona problem

Thomas Alva Whitehurst, An application of orthogonal polynomials to random walks ..............................

David J. Winter, Root locologies and idempotents of Lie and nonassociative algebras

William Robin Zame, The classification of uniform algebras on plane domains 